\title{
CINÉTICA E TERMODINÂMICA DA ADSORÇÃO DE CAROTENÓIDES E CLOROFILAS NO BRANQUEAMENTO DE ÓLEO DE FARELO DE ARROZ
}

\author{
R. S. POHNDORF ${ }^{1 *}$, F. F. YOUNAN ${ }^{1}$, J. F. COSTA $^{1}$, B. S. FARIAS ${ }^{1}$, T. R. S. CADAVAL Jr ${ }^{1}$, L. $^{2}$ \\ A. A. PINTO ${ }^{1}$ \\ ${ }^{1}$ Universidade Federal do Rio Grande, Escola de Química e Alimentos \\ *e-mail: ricardoscherer.eng@gmail.com
}

\begin{abstract}
RESUMO
Neste trabalho, a adsorção de carotenóides e clorofilas no branqueamento de óleo de farelo de arroz (OFA) foi investigada em diferentes temperaturas $\left(100,110\right.$ e $\left.120^{\circ} \mathrm{C}\right) \mathrm{e}$ concentrações de terra ativada $(0,5-2,5 \% \mathrm{~m} / \mathrm{m})$. O estudo cinético revelou que a partir de 20 min a capacidade de adsorção tende ficar constante. O modelo de pseudo-primeira ordem foi mais adequado para descrever o comportamento cinético da adsorção de clorofilas, e o modelo de pseudo-segunda ordem ajustou-se satisfatoriamente aos dados experimentais para a adsorção de carotenóides. A elevação da temperatura de $100{ }^{\circ} \mathrm{C}$ para $120{ }^{\circ} \mathrm{C}$ aumentou $20 \%$ a capacidade máxima de adsorção de carotenóides e $35 \%$ a adsorção de clorofilas. O modelo de Freundlich foi adequado para representar os dados de equilíbrio para ambos os pigmentos. O branqueamento de OFA foi considerado um processo endotérmico, favorável e espontâneo. O calor isostérico da adsorção indicou que a superfície da terra ativada é heterogênea.
\end{abstract}

\section{INTRODUÇÃO}

A produção mundial de arroz (Oryza sativa L.) tem aumentado nos últimos anos, principalmente devido à expansão da área plantada e o aumento na produtividade. Além da casca (20\% do grão) que serve de biomassa para produção de energia em caldeiras, o farelo de arroz (8-11\% do grão) contém proteínas $(12-13 \% \mathrm{~m} / \mathrm{m})$, carboidratos (48$60 \% \mathrm{~m} / \mathrm{m})$ e lipídios $(18-25 \% \mathrm{~m} / \mathrm{m})$ (SILVA, SANCHES e AMANTE, 2006).

O óleo de farelo de arroz (OFA) apresenta características diferenciadas entre os óleos vegetais. Além de ser obtido a partir de um subproduto, ele possui alto teor de fitoquímicos e ácidos graxos mono e poliinsaturados. O óleo de farelo de arroz é uma das poucas fontes ricas em fitoquímicos bioativos comercialmente importantes e de interesse nutricional, farmacêutico e cosmético. Ele auxilia na redução do colesterol LDL devido ao seu perfil de ácidos graxos e o alto teor de compostos insaponificáveis. O óleo de farelo de arroz contém y-orizanol, que apresenta efeito antiinflamatório e protetor contra o estresse oxidativo, auxiliando no controle da diabete (KANAYA et al., 2004).

O refino químico é largamente aplicado nas indústrias de óleos vegetais, inclusive em OFA. Neste processo o óleo bruto é degomado, neutralizado, branqueado, decerado e desodorizado. Outra forma de purificar o óleo é através do refino físico, que tem sido avaliado no óleo de farelo de arroz para diminuir as perdas de $\mathrm{\gamma}$-orizanol durante o refino químico, especialmente na etapa de neutralização (PAUCAR-MENACHO et al., 2007).

A etapa de branqueamento é importante tanto para o refino químico quanto para o 
físico, uma vez que ela é responsável pela remoção de pigmentos, produtos de oxidação primária (peróxidos) e secundária (aldeídos, cetonas e alcoóis), além de ser a única etapa capaz de remover traços de metais que podem favorecer a oxidação do óleo (MONTE et al., 2015).

Assim, objetivo neste trabalho foi estudar influência da temperatura e da concentração de adsorvente no processo de branqueamento de óleo de farelo de arroz. A cinética de adsorção de carotenóides e clorofilas foi avaliada pelos modelos cinéticos de pseudo-primeira ordem, pseudo-segunda ordem e Elovich. As isotermas de equilíbrio foram obtidas utilizando diferentes concentrações de adsorventes, e as curvas foram ajustadas aos modelos de Langmuir e Freundlich. As variações dos parâmetros termodinâmicos energia livre de Gibbs $\left(\Delta \mathrm{G}^{0}\right)$, entalpia $\left(\Delta \mathrm{H}^{0}\right)$, entropia $\left(\Delta \mathrm{S}^{0}\right)$ e calor isostérico $\left(\Delta \mathrm{H}_{\mathrm{a}}\right)$ foram estimadas.

\section{MATERIAL E MÉTODOS}

O óleo de farelo de arroz neutralizado foi obtido em uma empresa de refino de óleo local, e acondicionado à $-20^{\circ} \mathrm{C}$ para evitar oxidação. Os experimentos foram realizados no Laboratório de Operações Unitárias da Escola de Química e Alimentos (EQA) da Universidade Federal do Rio Grande (FURG), localizada no sul do Brasil. Foi utilizada terra ativada (Tonsil Supreme 110 FF) como adsorvente comercial nos ensaios de branqueamento.

\subsection{Caracteríticas do adsorvente}

A terra ativada foi caracterizada por microscopia eletrônica de varredura (MEV) (Jeol, JSM-6060, Japão) e espectroscopia de energia dispersiva (EDS) (Jeol, JSM-5800, Japão). A área superficial do adsorvente foi determinada por um analisador de adsorção volumétrica (Quantachrome Instruments, New
Win 2, EUA) usando o método Brunauer, Emmet e Teller (BET).

\subsection{Determinação de carotenóides e clorofilas}

A determinação da concentração de carotenóides foi realizada por espectrofotometria (Quimis, Q108, Brasil). Amostras de OFA foram diluídas em hexano $(10 \% \mathrm{~m} / \mathrm{v})$ e filtradas. A concentração de carotenóides foi obtida por leituras de absorbância a $446 \mathrm{~nm}$ e expressa em mg kg-1 (Equação 1), de acordo com metodologia proposta por MPOB (2005).

$C=\frac{383 A_{446}}{L c}$

onde $C$ é a concentração de carotenóides (mg $\left.\mathrm{kg}^{-1}\right), A$ é a leitura de absorbância á $446 \mathrm{~nm}$, $L$ é o comprimento da cubeta $(\mathrm{cm}), c$ é a concentração da solução de óleo em hexano $\left(\mathrm{g} 100 \mathrm{~mL}^{-1}\right)$ e 383 é o coeficiente de extinção para carotenóides.

A concentração de clorofilas (predominantemente feofitina- $a$ ) foi determinada por absorbância nos comprimentos de onda 630, 670 e $710 \mathrm{~nm}$ (Sabah, 2007), e calculada pela Equação 2.

$C l=\frac{\left[A_{670}-\left(A_{360}+A_{710}\right) / 2\right] \mathrm{V}}{0,0964 M L}$

onde $\mathrm{Cl}$ é a concentração de clorofilas ( $\mathrm{mg} \mathrm{kg}^{-}$ $\left.{ }^{1}\right), A_{i}$ é a leitura de absorbância no comprimento de onda $i(\mathrm{~nm}), V$ é o volume de hexano $(\mathrm{mL}), L$ é a largura da cubeta $(\mathrm{cm})$ e $M$ é a massa de óleo $(\mathrm{g})$.

\subsection{Experimentos de adsorção}

Amostras (40 g) de OFA neutralizado foram aquecidas sob agitação constante (40 rpm) utilizando um agitador magnético com aquecimento. A taxa de aquecimento foi de 7 ${ }^{\circ} \mathrm{C} \min ^{-1}$, e o óleo foi mantido à pressão 
absoluta de $70 \mathrm{mmHg}$. Após atingir a temperatura desejada, o adsorvente foi adicionado ao óleo. As curvas cinéticas foram obtidas ao longo de $60 \mathrm{~min}$ utilizando $1 \%$ $\mathrm{m} / \mathrm{m}$ de terra ativada à $120 \pm 1{ }^{\circ} \mathrm{C}$. Para as curvas de equilíbrio foram utilizadas diferentes concentrações de adsorvente $(0,5$ $2,5 \% \mathrm{~m} / \mathrm{m})$, em diferentes temperaturas (100$\left.120^{\circ} \mathrm{C}\right)$. O tempo de contato entre $\mathrm{o}$ adsorvente e o óleo foi de $120 \mathrm{~min}$ (BAYRAK, 2003). O adsorvente foi separado imediatamente do óleo por centrifugação (3500 $\times g$ por $3 \mathrm{~min}$ ). Os parâmetros utilizados neste estudo foram baseados em testes preliminares e dados da literatura (RIBEIRO et al., 2001; SILVA et al., 2013). Os experimentos foram realizados em triplicata. As amostras foram analisadas quanto as concentrações de carotenóides e clorofilas. A capacidade de adsorção em qualquer tempo $\left(q_{t}\right)$ e no equilíbrio $\left(q_{e}\right)$ foram determinadas pelas Equações 3 e 4, respectivamente:

$$
\begin{aligned}
& q_{t}=\frac{M_{o}\left(C_{i}-C_{t}\right)}{M_{a}} \\
& q_{e}=\frac{M_{o}\left(C_{i}-C_{e}\right)}{M_{a}}
\end{aligned}
$$

onde $M_{o}$ é a quantidade de óleo $(\mathrm{kg}), M_{a}$ é a quantidade de adsorvente $(\mathrm{kg}) C_{i}$ é a concentração inicial do adsorbato $\left(\mathrm{mg} \mathrm{kg}^{-1}\right)$, $C_{t}$ e $C_{e}$ são as concentrações do adsorbato (mg $\mathrm{kg}^{-1}$ ) no tempo $t$ e no equilíbrio ( $t=120 \mathrm{~min}$ ), respectivamente.

\subsection{Modelos cinéticos e de equilíbrio}

O comportamento cinético da adsorção de pigmentos foi obtido por ajuste dos modelos de pseudo-primeira ordem (Equação 5), pseudo-segunda ordem (Equação 6) e Elovich (Equação 7). Estes modelos são baseados na capacidade de adsorção ao invés da concentração da solução

$$
\begin{aligned}
& q_{t}=q_{1}\left(1-\exp \left(-k_{1} t\right)\right) \\
& q_{t}=\frac{t}{\left(1 / k_{2} q_{2}\right)^{2}+\left(t / q_{2}\right)} \\
& q_{t}=\frac{1}{a}+\ln (1+a b t)
\end{aligned}
$$

onde $k_{1}$ e $k_{2}$ são as constantes da taxa de adsorção para os modelos de pseudo-primeira ordem $\left(\mathrm{min}^{-1}\right)$ e pseudo-segunda ordem $(\mathrm{kg}$ $\left.\mathrm{mg}^{-1} \mathrm{~min}^{-1}\right)$ respectivamente, $q_{1}$ e $q_{2}$ representam a capacidade máxima de adsorção teórica ( $\left.\mathrm{mg} \mathrm{kg}^{-1}\right)$ no tempo $t$ ( $\left.\min \right), a$ é o parâmetro do modelo de Elovich associado a velocidade inicial $\left(\mathrm{mg} \mathrm{kg}^{-1} \mathrm{~min}^{-1}\right)$ e $b$ é a constante de dessorção $\left(\mathrm{kg} \mathrm{mg}^{-1}\right)$.

$\mathrm{O}$ efeito da temperatura de branqueamento na constante da taxa de adsorção foi analisado pela relação de Arrhenius (Equação 8).

$k=A e^{-\frac{E a}{R T}}$

onde $k$ é a constante da taxa de adsorção associado a velocidade da adsorção $\left(\mathrm{min}^{-1}\right), A$ é o fator de frequência $\left(\mathrm{s}^{-1}\right), E a$ é a energia de ativação $\left(\mathrm{kJ} \mathrm{mol}^{-1}\right), \mathrm{R}$ é a constante universal dos gases $\left(8,314 \mathrm{~kJ} \mathrm{~mol}^{-1} \mathrm{~K}^{-1}\right)$ e $T$ é a temperatura absoluta $(\mathrm{K})$.

As isotermas de equilíbrio foram ajustadas pelos modelos de Langmuir e Freundlich. O modelo de Langmuir (Equação 9) assume que a adsorção ocorre em monocamada, onde a superfície do adsorvente é homogênea e os sítios ativos possuem a mesma afinidade e energia. A isoterma de Freundlich (Equação 10) assume que a superfície do adsorvente é heterogênea, e que a quantidade de adsorbato adsorvida aumenta infinitamente com a concentração (SILVA et al., 2013): 
$q_{e}=\frac{q_{m} k_{L} C_{e}}{1+\left(k_{L} C_{e}\right)}$

$$
q_{e}=k_{F} C_{e}^{1 / n F}
$$

onde $K_{F}$ é a constante de Freundlich ( $\mathrm{mg} \mathrm{kg}$ $1_{\text {adsorvente }}\left(\mathrm{mg} \mathrm{kg}^{-1}{ }_{\text {oleo }}\right)^{-1 / \mathrm{nF}}, 1 / n_{F}$ é o fator de heterogeneidade, $q_{m}$ é a capacidade máxima de adsorção ( $\mathrm{mg} \mathrm{kg}^{-1}$ adsorvente) e $K_{L}$ é a constante de Langmuir ( $\left.\mathrm{kg}_{\text {adsorvente }} \mathrm{mg}^{-1}\right)$.

\subsection{Avaliação dos parâmetros termodinâmicos}

A adsorção de pigmentos de OFA foi avaliada pelas variações de energia livre de Gibbs $\left(\Delta G^{0}, \mathrm{~kJ} \mathrm{~mol}^{-1}\right)$, de entalpia $\left(\Delta H^{0}, \mathrm{~kJ}\right.$ $\mathrm{mol}^{-1}$ ) e de entropia $\left(\Delta S^{0}, \mathrm{~kJ} \mathrm{~mol}^{-1} \mathrm{~K}^{-1}\right)$. Estes valores foram estimados por meio dos parâmetros obtidos no melhor ajuste das isotermas de acordo com as Equações 11 e 12 (MILONJIĆ, 2007).

$\Delta G^{0}=-R T \ln \left(k_{e}\right)$

$\frac{\Delta G^{0}}{R T}=\frac{\Delta H^{0}}{R T}-\frac{\Delta S^{0}}{R}$

onde $K_{e}$ é a constante de equilíbrio adimensional obtida a partir das isotermas linearizadas, $T$ é a temperatura absoluta $(\mathrm{K}) \mathrm{e}$ $R$ é a constante universal dos gases $(8,314 \mathrm{~kJ}$ $\mathrm{mol}^{-1} \mathrm{~K}$ ).

O calor envolvido na adsorção a uma área superficial constante ou calor isostérico $\left(\Delta H_{a}, \mathrm{~kJ} \mathrm{~mol}^{-1}\right)$ é um importante parâmetro termodinâmico utilizado para elucidar a natureza da adsorção de pigmentos e pode ser obtido a partir da Equação 13, derivada da equação de Clausius-Clapeyron (BAYRAK, 2003).

$\ln C_{e}=\frac{\Delta H_{a}}{R} \frac{1}{T}$

\subsection{Análise estatística}

Os modelos cinéticos foram ajustados aos dados experimentais através de regressão não linear usando o método Quasi-Newton. A qualidade do ajuste foi obtida pelo coeficiente de determinação $\left(R^{2}\right)$, coeficiente de determinação ajustado $\left(R^{2}{ }_{a j u .}\right)$ e erro médio relativo $(E M R)$. Os parâmetros foram obtidos pelo software Statistic 7.0 (StatSoft, EUA) e os gráfico construídos pelo software SigmaPlot 10.0 (Systat, EUA).

\section{RESULTADO E DISCUSSÃO}

\subsection{Características do adsorvente}

As terras ativadas são largamente utilizadas no branqueamento de óleo vegetais. Basicamente são argilominerais com superfície ativada por tratamento ácido e em sua composição possuem silicatos hidratados de alumínio, ferro e magnésio. $\mathrm{O}$ uso de bentonita, sepiolita e atapulgita tem sido reportado na literatura para a adsorção de pigmentos em óleos (LIU, HUANG e WANG, 2008). A Figura 1A apresenta uma imagem (MEV) da terra ativada utilizada neste estudo (Tonsil Supreme 110 FF). Observam-se partículas com diferentes formatos, superfície irregular e com diâmetro predominantemente inferior a $50 \mu \mathrm{m}$. A composição elementar mostrada no espectro EDS (Figura 1B) indica em percentual mássico a presença de silício $(51,32 \pm 8,81 \%)$, alumínio $\quad(13,26 \pm 2,72 \%)$ oxigênio $(22,21 \pm 3,27 \%)$, ferro $(5,28 \pm 1,67 \%)$ e magnésio $(2,02 \pm 0,52 \%)$, além de outros elementos em menor concentração. $\mathrm{O}$ alto teor de silicatos no adsorvente tende a melhorar a capacidade de branqueamento. A área superficial determinada por BET para terra ativada foi de $240,1 \pm 5,9 \mathrm{~m}^{2} \mathrm{~g}^{-1}$. Didi et al. (2009) caracterizou adsorventes comerciais utilizados no branqueamento de óleos vegetais, indicando que a área superficial varia entre 165 e $340 \mathrm{~m}^{2} \mathrm{~g}^{-1}$. A área superficial depende principalmente da intensidade do tratamento ácido empregado, e 
é proporcional a capacidade de branqueamento do óleo. A ativação ácida proporciona uma substituição de cátions por prótons. Os íons metálicos $\left(\mathrm{Fe}^{+2}, \mathrm{Mg}^{+2}\right.$ e $\left.\mathrm{Al}^{+3}\right)$ são substituídos por prótons que constituem centros ativos de interação entre a partícula adsorvente e as moléculas adsorvidas (CHRISTIDIS, SCOTT e DUNHAM, 1997).

Figura 1 - Características de terra ativada comercial: (A) imagem de MEV e (B) espectro EDS
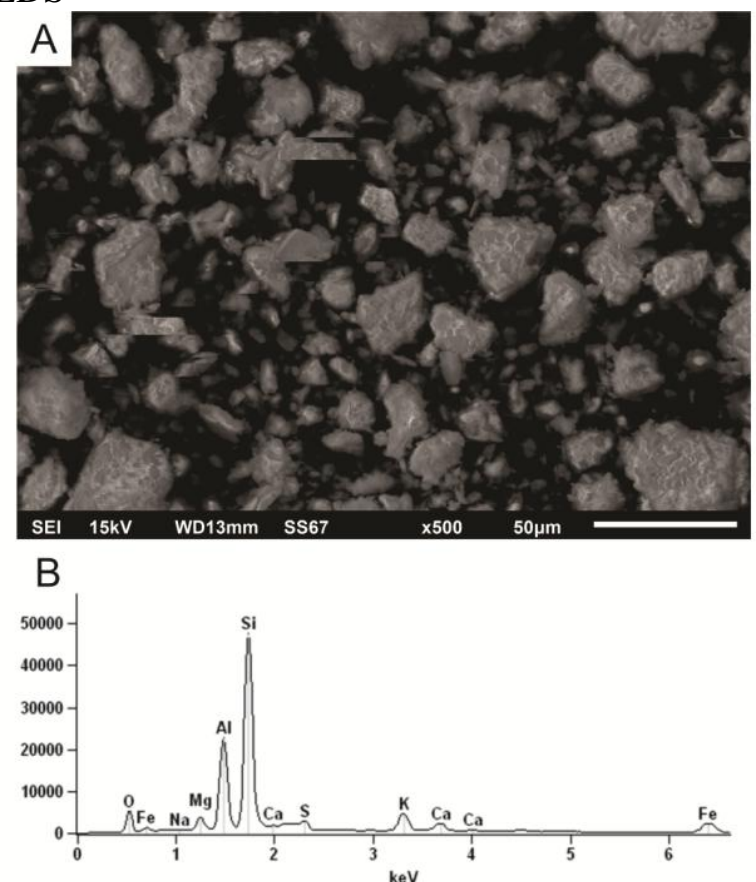

Fonte: Autor (2015).

\subsection{Estudo cinético}

A Figura 2 apresenta as curvas cinéticas da adsorção de carotenóides de OFA em diferentes temperaturas $\left(100,110\right.$ e $\left.120{ }^{\circ} \mathrm{C}\right)$ utilizando $1 \% \quad(\mathrm{~m} / \mathrm{m})$ de adsorvente em relação ao óleo. A adsorção de carotenóides ocorre rapidamente nos primeiros $10 \mathrm{~min}$ do processo, onde 69, 77 e $87 \%$ da capacidade máxima de adsorção foram alcançadas para as temperaturas de 100,110 e $120{ }^{\circ} \mathrm{C}$, respectivamente. Após 10 min de branqueamento a taxa de adsorção de carotenóides diminui drasticamente, tendendo a estabilizar após $30 \mathrm{~min}$. O aumento da temperatura de 100 para $120{ }^{\circ} \mathrm{C}$ proporcionou um ganho de $20 \%$ na capacidade máxima de adsorção de carotenóides. Embora altas temperaturas aumentem a taxa de adsorção, podem também causar degradação de compostos menores, hidrólise de ácidos graxos e autoxidação no óleo. Neste sentido, a temperatura máxima de branqueamento depende das características de cada óleo. Ma e Lin (2004) sugerem que em temperaturas menores do que $100{ }^{\circ} \mathrm{C}$, a adsorção é comandada por forças de van der Walls (fisissorção), e que o aumento da temperatura favorece a adsorção de $\beta$-caroteno tanto nas camadas mais externas quanto nos poros do adsorvente.

Figura 2 - Influência da temperatura na cinética da adsorção de carotenóides de óleo de farelo de arroz

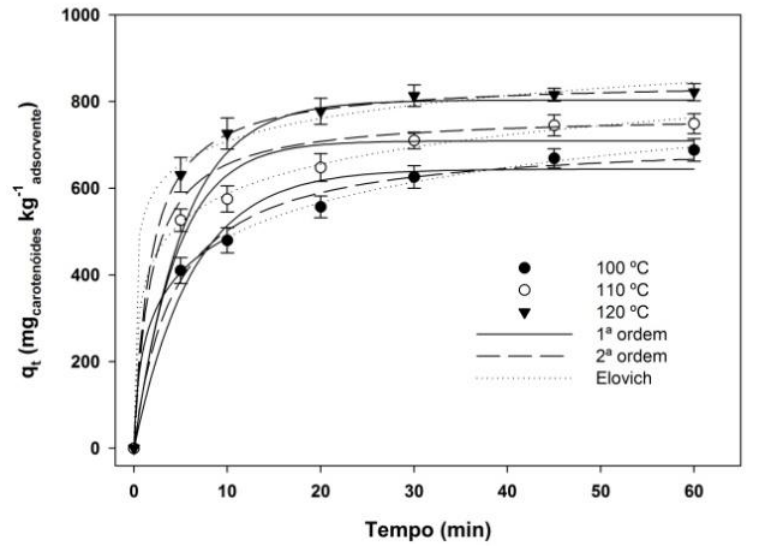

Fonte: Autor (2015).

As curvas cinéticas da adsorção de clorofilas são apresentadas na Figura 3. O comportamento da adsorção de clorofilas foi semelhante ao apresentado na adsorção de carotenóides, com uma elevada taxa de adsorção inicial e progressiva diminuição até o final do processo. A capacidade de adsorção de clorofilas foi maior do que a de carotenóides em $60 \mathrm{~min}$. $\mathrm{O}$ aumento da temperatura de 100 para $120{ }^{\circ} \mathrm{C}$ aumentou $35 \%$ a capacidade máxima de adsorção de clorofilas. De acordo com Tong et al. (2008), as clorofilas são adsorvidas e alcançam o 
equilíbrio mais rapidamente na superfície da terra ativada indicando maior interação química entre o adsorvente e o adsorbato.

Figura 3 - Influência da temperatura na cinética da adsorção de clorofilas de OFA

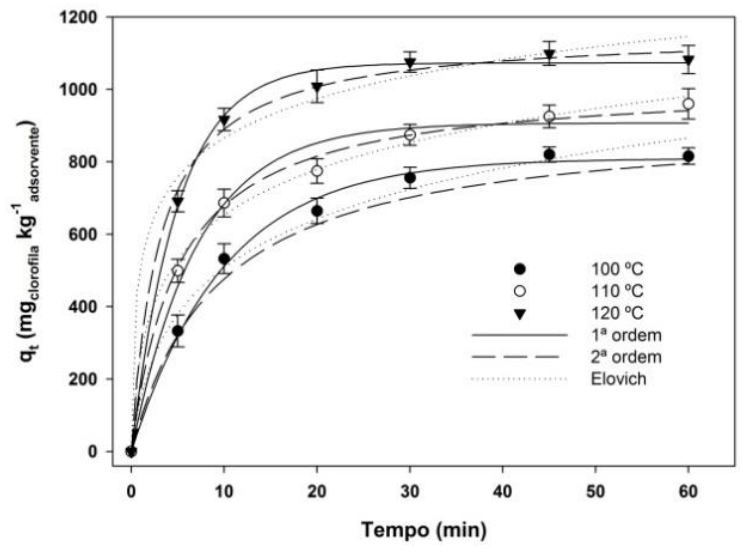

Fonte: Autor (2015).

Os modelos de pseudo-primeira ordem, pseudo-segunda ordem e Elovich foram ajustados às curvas cinéticas experimentais. Os resultados são apresentados na Tabela 1. Baseado nos maiores valores de coeficiente de determinação $\left(R^{2}\right), \quad$ coeficiente de determinação ajustado $\left(R_{\text {aju }}^{2}\right)$ e nos menores valores de erro médio relativo $(E M R)$, pode ser afirmado que os modelos mais adequados para representar a cinética de adsorção de carotenóides e clorofilas foram respectivamente, os modelos de pseudosegunda ordem e pseudo-primeira ordem. Entretanto o modelo de Elovich apresentou um bom ajuste, tanto para carotenóides quanto para clorofilas, uma vez que o modelo é utilizado em sistemas heterogêneos.

A constante da taxa de adsorção $\left(k_{1}\right)$ está diretamente relacionada com a velocidade da adsorção. Neste respeito, os carotenóides foram adsorvidos mais rapidamente do que as clorofilas, entretanto os valores foram próximos. $\mathrm{O}$ aumento na temperatura do óleo de 100 para $120{ }^{\circ} \mathrm{C}$ duplicou a constante da taxa de adsorção $\left(k_{1}\right)$ para ambos os pigmentos. Resultados semelhantes foram encontrados por Silva et al. (2013), onde ambos os modelos de pseudoprimeira ordem e pseudo-segunda ordem foram adequados para descrever 0 comportamento cinético de branqueamento de óleo de palma. Tong et al. (2008) indicou o modelo de pseudo-segunda ordem foi capaz de descrever satisfatoriamente a adsorção de carotenóides e clorofilas por bentonita ativada. Outros modelos também são utilizados na literatura para adsorção de pigmentos em óleos (MONTE et al., 2015). A energia de ativação para adsorção de carotenóides foi de $36,25 \pm 3,68 \mathrm{~kJ} \mathrm{~mol}^{-1}$ e para adsorção de clorofila foi de $42,21 \pm 2,83 \mathrm{~kJ}$ $\mathrm{mol}^{-1}$, indicando que as clorofilas necessitaram um pouco mais de energia para serem adsorvidas.

\subsection{Estudo de equilíbrio}

As isotermas de equilíbrio foram obtidas em diferentes temperaturas $\left(100,110\right.$ e $\left.120^{\circ} \mathrm{C}\right) \mathrm{e}$ diferentes concentrações de adsorventes, no óleo de farelo de arroz $(0,5-2,5 \% \mathrm{~m} / \mathrm{m})$. As curvas para adsorção de carotenóides e clorofilas são apresentadas na Figura 4 e na Figura 5, respectivamente. A capacidade de adsorção aumentou com a elevação da temperatura, para ambos os pigmentos. Isto pode ser atribuído pela ativação de mais sítios de adsorção na terra ativada com o aumento da temperatura. Embora exista, o efeito da temperatura na adsorção é menos pronunciado do que o da concentração de adsorvente. O mesmo comportamento foi encontrado por Boki et al. (1992) no branqueamento de diferentes óleos vegetais.

A concentração inicial de carotenóides em OFA foi de $13,88 \pm 0,99 \mathrm{mg} \mathrm{kg}^{-1} \mathrm{e}$ de clorofilas foi de $14,10 \pm 0,70 \mathrm{mg} \mathrm{kg}^{-1}$. Assim, pode-se notar que os pigmentos competem entre si no processo de adsorção (SABAH, 2007). As clorofilas demonstraram mais afinidade com a terra ativada do que os carotenóides, conforme indicado pelos valores da capacidade de adsorção. Um comportamento semelhante foi observado por 
Tabela 1 - Parâmetros cinéticos da adsorção de pigmentos de óleo de farelo de arroz.

\begin{tabular}{|c|c|c|c|c|c|c|}
\hline \multirow{2}{*}{$\begin{array}{l}\text { Adsorbato } \\
\text { Temperatura }\left({ }^{\circ} \mathrm{C}\right)\end{array}$} & \multicolumn{3}{|l|}{ Carotenóides } & \multicolumn{3}{|l|}{ Clorofilas } \\
\hline & 100 & 110 & 120 & 100 & 110 & 120 \\
\hline \multicolumn{7}{|l|}{ Pseudo-primeira ordem } \\
\hline$q_{1}\left(\mathrm{mg} \mathrm{kg}^{-1}\right)$ & $644 \pm 6$ & $709 \pm 9$ & $803 \pm 19$ & $809 \pm 13$ & $907 \pm 3$ & $1073 \pm 22$ \\
\hline$k_{l}\left(\min ^{-1}\right)$ & $0,16 \pm 0,01$ & $0,22 \pm 0,01$ & $0,29 \pm 0,01$ & $0,10 \pm 0,01$ & $0,14 \pm 0,01$ & $0,20 \pm 0,01$ \\
\hline$R^{2}$ & 0,968 & 0,972 & 0,995 & 0,995 & 0,983 & 0,997 \\
\hline$R_{a j u .}^{2}$ & 0,957 & 0,963 & 0,993 & 0,993 & 0,977 & 0,996 \\
\hline$A R E(\%)$ & 7,30 & 6,30 & 2,52 & 3,03 & 4,69 & 1,90 \\
\hline \multicolumn{7}{|l|}{ Pseudo-segunda ordem } \\
\hline$q_{2}\left(\mathrm{mg} \mathrm{kg}^{-1}\right)$ & $714 \pm 10$ & $770 \pm 12$ & $848 \pm 21$ & $919 \pm 15$ & $1020 \pm 11$ & $1160 \pm 18$ \\
\hline$k_{2} \times 10^{3}\left(\mathrm{~kg} \mathrm{mg}^{-1} \mathrm{~min}^{-1}\right)$ & $0,332 \pm 0,021$ & $0,743 \pm 0,025$ & $0,689 \pm 0,036$ & $0,117 \pm 0,016$ & $0,195 \pm 0,010$ & $0,284 \pm 0,022$ \\
\hline$R^{2}$ & 0,992 & 0,992 & 0,999 & 0,979 & 0,997 & 0,997 \\
\hline$R_{a j u .}^{2}$ & 0,989 & 0,989 & 0,999 & 0,972 & 0,996 & 0,996 \\
\hline$A R E(\%)$ & 3,59 & 3,31 & 0,49 & 6,02 & 1,86 & 2,13 \\
\hline \multicolumn{7}{|l|}{ Elovich } \\
\hline$a \times 10^{3}\left(\mathrm{mg} \mathrm{kg}^{-1} \mathrm{~min}^{-1}\right)$ & $8,46 \pm 0,11$ & $10,22 \pm 0,56$ & $13,20 \pm 0,88$ & $4,78 \pm 0,40$ & $5,39 \pm 0,20$ & $6,39 \pm 0,45$ \\
\hline$b\left(\mathrm{~kg} \mathrm{mg}^{-}\right)$ & $707 \pm 55$ & $3952 \pm 102$ & $87254 \pm 3256$ & $215 \pm 13$ & $607 \pm 15$ & $3902 \pm 158$ \\
\hline$R^{2}$ & 0,998 & 0,998 & 0,996 & 0,987 & 0,995 & 0,985 \\
\hline$R_{a j u .}^{2}$ & 0,997 & 0,997 & 0,995 & 0,983 & 0,993 & 0,980 \\
\hline$A R E(\%)$ & 1,35 & 1,65 & 2,46 & 5,86 & 2,71 & 4,70 \\
\hline
\end{tabular}

Fonte: Autor (2015).

Figura 4 - Curvas de equilíbrio para adsorção de carotenóides de OFA

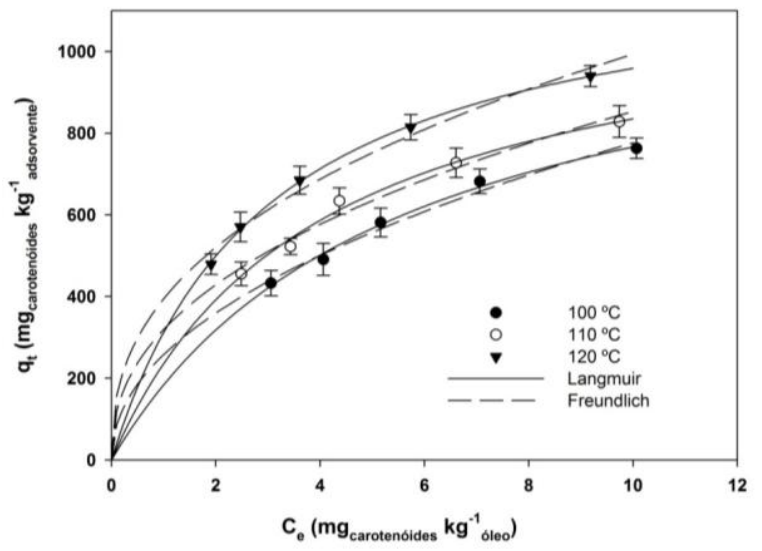

Fonte: Autor (2015).

Ribeiro et al. (2001) estudando a adsorção de pigmentos e produtos de oxidação em miscela de óleo de oliva com hexano. Na temperatura de $120{ }^{\circ} \mathrm{C}$, o emprego de $1 \%(\mathrm{~m} / \mathrm{m})$ de terra ativada causou uma intensa diminuição no teor de clorofilas, enquanto que a diminuição no teor de carotenóides foi menos pronunciada.

Figura 5 - Curvas de equilíbrio para adsorção de clorofilas de OFA por terra ativada

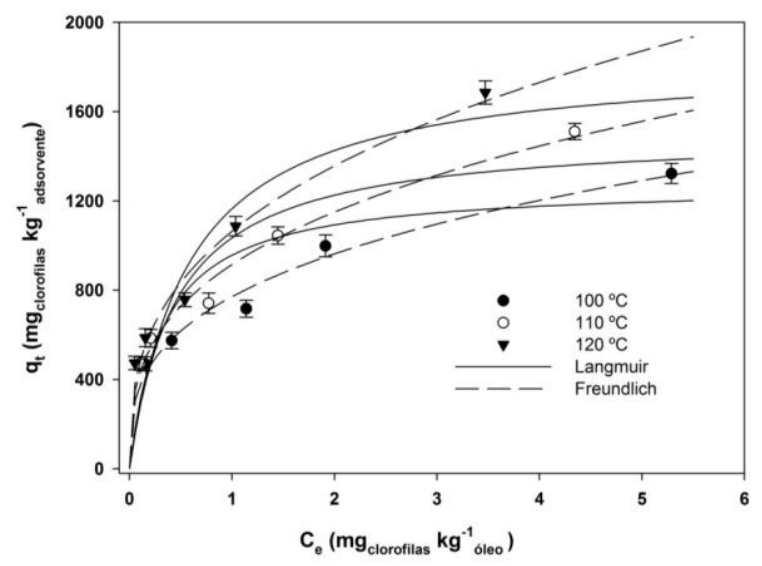

Fonte: Autor (2015).

As curvas experimentais de equilíbrio foram ajustadas aos modelos de Langmuir e 
Freundlich, e os resultados são apresentados na Tabela 2. Ambos os modelos foram adequados para descrever o processo de adsorção de carotenóides, em diferentes temperaturas. Entretanto o modelo de Freundlich apresentou melhor ajuste aos dados experimentais da adsorção de clorofilas do que o modelo de Langmuir, evidenciado pelos altos valores de coeficiente de determinação $\left(R^{2}\right), \quad$ coeficiente de determinação ajustado $\left(R^{2}\right.$ aju $)$ e pelos menores valores do erro médio relativo (EMR). Estes resultados estão coerentes com os apresentados na literatura (BAYRAK, 2008;
LIU, HUANG e WANG, 2008), uma vez que o modelo de Langmuir assume que a superfície do adsorvente é homogênea, enquanto que o modelo de Freundlich se baseia na dependência da energia dos sítios de adsorção. O fator de heterogeneidade do modelo de Freundlich $(1 / n)$ apresentou valores entre 2 e 4 indicando alta afinidade entre o adsorbato e o adsorvente e mostrou que o processo de adsorção foi favorável. Os maiores valores da constante de Freundlich $\left(k_{\mathrm{F}}\right)$ demonstraram que a adsorção foi mais efetiva na temperatura de $120{ }^{\circ} \mathrm{C}$.

Tabela 2 - Parâmetros de equilíbrio para a adsorção de pigmentos de óleo de farelo de arroz.

\begin{tabular}{|c|c|c|c|c|c|c|}
\hline \multirow{2}{*}{$\begin{array}{l}\text { Adsorbato } \\
\text { Temperatura }\left({ }^{\circ} \mathrm{C}\right)\end{array}$} & \multicolumn{3}{|l|}{ Carotenóides } & \multicolumn{3}{|l|}{ Clorofilas } \\
\hline & 100 & 110 & 120 & 100 & 110 & 120 \\
\hline \multicolumn{7}{|l|}{ Modelo de Langmuir } \\
\hline$q_{m}\left(\mathrm{mg} \mathrm{kg}_{\text {ads }}^{-1}\right)$ & $1183 \pm 62$ & $1164 \pm 70$ & $1245 \pm 72$ & $1273 \pm 52$ & $1503 \pm 80$ & $1838 \pm 95$ \\
\hline$K_{L}\left(\mathrm{~kg}_{\text {oil }} \mathrm{mg}^{-1}\right)$ & $0,184 \pm 0,008$ & $0,254 \pm 0,007$ & $0,335 \pm 0,020$ & $3,030 \pm 0,042$ & $2,208 \pm 0,095$ & $1,730 \pm 0,129$ \\
\hline$R^{2}$ & 0,993 & 0,990 & 0,999 & 0,870 & 0,822 & 0,786 \\
\hline$R_{a d j}^{2}$ & 0,991 & 0,987 & 0,999 & 0,827 & 0,763 & 0,715 \\
\hline$A R E(\%)$ & 1,59 & 1,73 & 0,76 & 13,62 & 21,45 & 28,67 \\
\hline \multicolumn{7}{|l|}{ Modelo de Freundlich } \\
\hline$k_{F}\left(\mathrm{mg} \mathrm{kg}^{-1}{ }_{\text {ads }}\right)\left(\mathrm{mg} \mathrm{kg}^{-1}{ }_{\text {oil }}\right)^{-1 / n}$ & $258 \pm 8$ & $319 \pm 6$ & $394 \pm 11$ & $771,3 \pm 11$ & $915 \pm 12$ & $1064 \pm 20$ \\
\hline $1 / n_{F}$ & $2,09 \pm 0,07$ & $2,34 \pm 0,09$ & $2,49 \pm 0,05$ & $3,12 \pm 0,02$ & $3,02 \pm 0,01$ & $2,85 \pm 0,03$ \\
\hline$R^{2}$ & 0,981 & 0,998 & 0,982 & 0,974 & 0,981 & 0,965 \\
\hline$R_{a d j}^{2}$ & 0,975 & 0,997 & 0,976 & 0,965 & 0,974 & 0,953 \\
\hline$A R E(\%)$ & 2,61 & 3,18 & 3,14 & 5,62 & 6,22 & 9,82 \\
\hline
\end{tabular}

Fonte: Autor (2015).

\subsection{Resultados termodinâmicos}

Através dos parâmetros termodinâmicos, a adsorção de pigmentos de OFA foi avaliada pela constante de equilíbrio $\left(k_{\mathrm{e}}\right)$, e pelas variações de energia livre de Gibbs $\left(\Delta G^{0}\right)$, de entalpia $\left(\Delta H^{0}\right)$ e de entropia $\left(\triangle S^{0}\right)$. Os resultados são mostrados na Tabela 3.

Pode ser observado na Tabela 3 que a constante de equilíbrio $\left(k_{\mathrm{e}}\right)$ aumenta com a elevação da temperatura, indicando a adsorção de pigmentos de OFA foi favorável com o aumento da temperatura. Os valores negativos de $\Delta G^{0}$ revelam que o processo foi espontâneo. Baseado nos valores positivos de $\Delta H^{0}$ é possível inferir que o processo foi endotérmico. De acordo com Bairak (2003) valores de $\Delta H^{0}$ acima de $20 \mathrm{~kJ} \mathrm{~mol}^{-1}$ indicam que $o$ processo é governado predominantemente por interações químicas.

Os valores positivos de $\Delta S^{0}$ indicam que a desordem na interface sólido-líquido aumenta durante o processo de adsorção. $\mathrm{O}$ calor isostérico de adsorção $\left(\Delta \mathrm{H}_{\mathrm{a}}\right)$ para diferentes concentrações de adsorventes, ou seja, comparado em relação a mesma área superficial é apresentado na Figura 6. 
Tabela 3 - Parâmetros termodinâmicos para a adsorção de pigmentos de óleo de farelo de arroz.

\begin{tabular}{lllll}
\hline Temperatura $\left({ }^{\circ} \mathrm{C}\right)$ & $k_{\mathrm{e}}$ & $\Delta G^{0}\left(\mathrm{~kJ} \mathrm{~mol}^{-1}\right)^{\mathrm{a}}$ & $\Delta H^{0}\left(\mathrm{~kJ} \mathrm{~mol}^{-1}\right)^{\mathrm{a}}$ & $\Delta S^{0}\left(\mathrm{~kJ} \mathrm{~mol}^{-1} \mathrm{~K}^{-1}\right)^{\mathrm{a}}$ \\
\hline Carotenóides & & & & \\
100 & $251 \pm 7$ & $-17,13 \pm 0,21$ & $25,42 \pm 4,44$ & $0,11 \pm 0,01$ \\
110 & $309 \pm 7$ & $-18,25 \pm 0,09$ & & \\
120 & $381 \pm 17$ & $-19,41 \pm 0,19$ & & \\
Clorofilas & & & & \\
100 & $772 \pm 12$ & $-20,62 \pm 0,07$ & $19,38 \pm 2,56$ & \\
110 & $914 \pm 15$ & $-21,71 \pm 0,11$ & & \\
120 & $1061 \pm 22$ & $-22,76 \pm 0,15$ & & \\
\hline
\end{tabular}

${ }^{a}$ Média \pm desvio padrão $(n=3)$.

Fonte: Autor (2015).

Figura 6 - Calor isostérico da adsorção de pigmentos em OFA

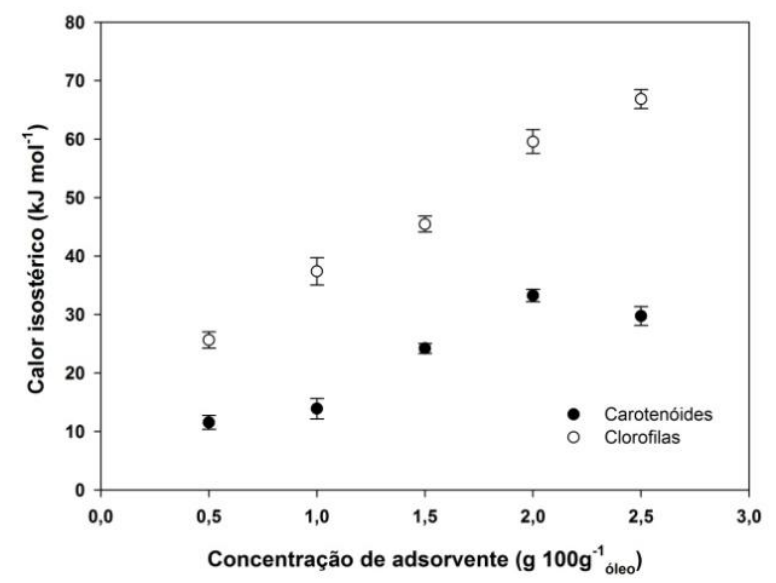

Fonte: Autor (2015).

É possível observar que os menores valores de $\Delta H_{a}$ são encontrados na menor concentração de adsorvente, para ambos os pigmentos. Isto ocorre devido ao carregamento da superfície do adsorvente. Aumentando a concentração do adsorvente haverá um ganho de área e os pigmentos serão adsorvidos preferencialmente nos sítios com maior energia. À medida que se diminui a concentração do adsorvente, os pigmentos são adsorvidos pelos sítios mais fracos energeticamente resultando em valores menores de $\Delta H_{a}$ (SILVA et al., 2013).

\section{CONCLUSÃO}

Neste trabalho foi avaliada a adsorção de carotenóides e clorofilas de óleo de farelo de arroz utilizando terra ativada como adsorvente. A caracterização do adsorvente revela que o mesmo é composto predominantemente por silicatos e apresenta elevada área superficial. As curvas cinéticas indicam que após $20 \mathrm{~min}$ de processo a taxa de adsorção diminui consideravelmente tendendo ao equilíbrio. Os modelos de pseudo-segunda ordem e pseudo-primeira ordem foram apropriados para representar a adsorção de carotenóides e clorofilas, respectivamente. A temperatura de $120{ }^{\circ} \mathrm{C}$ aumentou a capacidade de adsorção de pigmentos de OFA e o teor de clorofilas reduziu drasticamente utilizando $1 \%(\mathrm{~m} / \mathrm{m})$ de adsorvente. $\mathrm{O}$ modelo de Freundlich foi $\mathrm{o}$ mais adequado para descrever as curvas de equilíbrio do branqueamento. Os parâmetros termodinâmicos revelaram que o processo foi endotérmico, favorável e espontâneo. $\mathrm{O}$ calor isostérico da adsorção indicou que a superfície da terra ativada é heterogênea.

\section{REFERÊNCIAS}

BAYRAK, Y. Adsorption Isotherms in Bleaching Hazelnut Oil. Journal of the American Oil Chemists' Society, v.80, p.1143-1146, 2003. 
BOKI, K.; KUBO, M.; KAWASAKI, N.; MORI, H. Adsorption isotherms of pigments from alkali-refined vegetable oils with clay minerals. Journal of the American Oil Chemists' Society, v.69, p.372-378, 1992.

CHRISTIDIS, G. E.; SCOTT, P. W.; DUNHAM, A. C. Acid activation and bleaching capacity of bentonites fromthe islands of Milos and Chios, Agean, Greece. Applied Clay Science, v.12, p.329-347, 1997.

DIDI, M. A.; MAKHOUKHI, B.; AZZOUZ, A.; VILLEMIN, D. Colza oil bleaching through optimized acid activation of bentonite. A comparative study. Applied Clay Science, v.42, p.336-344, 2009.

KANAYA, Y.; DOI, T.; SASAKI, H.; FUJITA, A.; MATSUNO, S.; OKAMOTO, $\mathrm{K}$. Rice bran extract prevents the elevation of plasma peroxylipid in KKAy diabetic mice. Diabetes Research and Clinical Practice, v. 66, p.157-160, 2004.

LIU, Y.; HUANG, J. WANG, X. Adsorption isotherms for bleaching soybean oil with activated attapulgite. Journal of the American Oil Chemists' Society, v.85, p.979-984, 2008.

MA, M-H.; LIN, C-I. Adsorption kinetics of $\beta$-carotene from soy oil using regenerated clay. Separation and Purification Technology, v.39, p.201-209, 2004.

MILONJIĆ, S. K. A consideration of the correct calculation of thermodynamic parameters of adsorption. Journal of Serbian Chemistry Society, v.72, p.1363-1367, 2007.

MONTE, M. L.; MONTE, M. L.; POHNDORF, R. S.; CREXI, V. T.; PINTO, L. A. A. Bleaching with blends of bleaching earth and activated carbon reduces color and oxidation products of carp oil. European Journal of Lipid Science and Technology, v.117, p. 829-836, 2015.

MPOB. Malaysian Palm Oil Board. Determination of Carotene Content. Method n'. p2.6., p.194-197, 2005.

PESTANA-BAUER, V. R.; ZAMBIAZI, R. C.; MENDONÇA, C. R. B.; BENEITOCAMBRA, M.; RAMIS-RAMOS, G. $\mathrm{\gamma}$ oryzanol and tocopherol contents in residues of rice bran oil refining. Food Chemistry, v.134, p.1479-1483, 2012.

RIBEIRO, M. H. L.; LOURENÇO, P. A. S.; MONTEIRO, J. P.; FERREIRA-DIAS, S. Kinetics of selective adsorption of impurities from a crude vegetable oil in hexane to activated earths and carbons. European Food Resource Technology, v.213, p.132-138, 2001.

SABAH, E. Decolorization of vegetable oils: Chlorophyll- $a$ adsorption by acid-activated sepiolite. Journal of Colloid Interface Science, v.310, p1-7, 2007.

SILVA, M. A.; SANCHES, C.; AMANTE, E. $R$. Prevention of hydrolytic rancidity in rice bran. Journal of Food Engineering, v.75, p.487-491, 2006.

SILVA, S. M.; SAMPAIO, K. A.; CERIANI, R.; VERHÉ, R.; STEVENS, C.; DE GREYT, W.; MEIRELLES, A. J. A. Adsorption of carotenes and phosphorus from palm oil onto acid activated bleaching earth: Equilibrium, kinetics and thermodynamics. Journal of Food Engineering, v.118, p.341-349, 2013.

TONG, J.; WU, Z.; SUN, X.; XU, X.; LI, C. Adsorption Kinetics of $\beta$-Carotene and Chlorophyll onto Acid-activated. Chinese Journal of Chemical Engineering, v.16, p.270-276, 2008. 